\title{
A Comparison of Fluro-Deoxy Glucose-Positron Emission Tomography (FDG- PET) Versus Magnetic Resonance (MR) Based Target Volume Delineation in Post-operative Glioblastoma
}

\author{
Tejinder Kataria $^{1^{*}}$, Kuldeep Sharma ${ }^{1}$, Pranav Chadha ${ }^{2}$, Dhanraj Jangid ${ }^{3}$ and Kulbir Ahlawat ${ }^{4}$ \\ ${ }^{1}$ Division of Radiation Oncology, Medanta Cancer Institute, Medanta- The Medicity, Gurgaon, India \\ ${ }^{2}$ Radiation Oncology, Kokilaben Hospital, Mumbai, India \\ ${ }^{3}$ Department of Nuclear Medicine, Medanta- The Medicity, Gurgaon, India \\ ${ }^{4}$ Department of Radiology and Imaging, Medanta- The Medicity, Gurgaon, India
}

"Corresponding author: Tejinder Kataria, Division of Radiation Oncology, Medanta Cancer Institute, Medanta- The Medicity, Gurgaon-122001, India, Tel: 91119899407442; E-mail: teji1960@gmail.com

Received date: Nov 19, 2014, Accepted date: Dec 19, 2014, Publication date: Dec 23, 2014

Copyright: (c) 2014 Kataria T, et al. This is an open-access article distributed under the terms of the Creative Commons Attribution License, which permits unrestricted use, distribution, and reproduction in any medium, provided the original author and source are credited.

\begin{abstract}
Background: Glioblastoma is the commonest brain tumor of adults carrying a very poor prognosis. Targeting the tumor accurately during surgery and radiotherapy holds promise in future. PET with various tracers is being explored worldwide to enhance the accuracy of target delineation. This study was undertaken to evaluate the differences and correlation between the target volumes delineated by using MRI and 18F-FDG PET during RT planning and to evaluate whether inclusion of PET is helpful in better coverage of high risk area for recurrence.
\end{abstract}

Materials and Methods: Fifteen post-operative patients of glioblastoma were prospectively enrolled. Planning images were acquired with CT, MRI and delayed PET. Image fusion was done to delineate MR-based (GTV-MR, CTV-MR, PTV-MR), PET-based (GTV-PET, CTV-PET) and combined (GTV-X, CTV-X) volumes. Only MR-based volume was used for actual treatment. Mean volumes were calculated for each group for volumetric analysis.

Concordance Index $(\mathrm{Cl})$ was calculated for GTV and CTV as $\mathrm{Cl}=[(\mathrm{M}+\mathrm{P}) / \mathrm{X}]-1$, to find correlation between volumes, such that full concordance and full non-concordance between MRI and PET volumes would yield a value of 1 and 0 respectively.

On recurrence, the recurrent volumes ( $\mathrm{rVol}$ ) were marked and correlated with initial CTV-MR, CTV-PET and CTV$X$ to calculate the proportion of rVol covered within these volumes.

Observation and Results: Mean GTV-MR, GTV-PET and GTV-X were $84.4 \mathrm{cc}, 11 \mathrm{cc}$ and $89.2 \mathrm{cc}$, respectively. Median follow-up was 16.6 months for the overall group and 26.1 months for the surviving patients. Overall 1,2 years survival was $80 \%$ and $20 \%$ respectively.

Conclusion: Inclusion of PET-based abnormality while delineating the target volumes for glioblastoma leads to a non-significant increase in target volumes with better coverage of the high-risk region. Thus, targeting the common volume (CTV-X) for treatment may prove beneficial in avoiding marginal recurrences in glioblastoma.

Keywords: FDG-PET; Glioblastoma; MRI; Radiotherapy; Target delineation

\section{Introduction}

Glioblastoma is the commonest primary tumour of adult brain carrying an exceptionally poor prognosis [1]. The mean age at diagnosis is 61 years and males are more frequently affected than females, with a sex ratio of 3:2 [2]. Over the last few decades, radiotherapy (RT) has been shown to prolong survival in glioblastoma patients but unfortunately these patients progress within the treated field [3-5]. Using traditional dose of 6000 centiGray (cGy), approximately $80 \%$ of failures occur "centrally," i.e. within $2 \mathrm{~cm}$ of the gross tumor $[5,6]$. Despite dose escalation trials, local failure in the high-dose region continues to be the primary mode of failure [7].
Addition of concurrent and adjuvant temozolomide (TMZ) resulted in improved 2-year survival of newly diagnosed malignant glioma (mainly glioblastomas) from $11 \%$ to $27 \%$, and 5 -year survival from $2 \%$ to $10 \%$ but patients still continue to develop local failures [8]. Thus, there is a huge unmet need for further intensification of local therapy in conjunction with TMZ.

Modern RT techniques like stereotactic radio-surgery (SRS), stereotactic fractionated radiotherapy (SFRT), intensity-modulated radiotherapy (IMRT), and proton therapy have potential to precisely treat the targets to a higher doses but for this to succeed, the first requirement is to define the correct tumor boundries. Currently, magnetic resonance imaging (MRI) is the gold standard in defining target volumes for brain tumors. The area of enhancement on T1weighted (T1W) MRI is used to define the gross tumor volume (GTV) although it is not a fool-proof methodolgy. Several studies have shown 
Citation: Takeda K, Dobashi S, Komori S, Chida K, Kadoya N, et al. (2014) A Comparison of Fluro-Deoxy Glucose-Positron Emission Tomography (FDG-PET) Versus Magnetic Resonance (MR) Based Target Volume Delineation in Post-operative Glioblastoma. J Nucl Med Radiat Ther 6: 205. doi:10.4172/2155-9619.1000205

Page 2 of 10

that contrast enhancement on MRI may not represent the exact tumor boundaries as infiltrating glioma cells can be identified beyond the enhancing area [9]. Contrast enhancement is a nonspecific sign of blood-brain barrier disruptions [10] and is influenced by multiple factors like changing steroid dose, antiangiogenic agents, changing radiologic techniques etc. [11]. Moreover, enhancement can be induced by treatment-related inflammation, seizure activity, postsurgical changes, ischemia, subacute radiation effects, and radiation necrosis [12]. Thus, it is imperative to develop imaging methods that view tumor entirely and measure molecular pathologic processes within it [13].

Positron Emission Tomography (PET), using diverse radio-labelled compounds is being used for over two decades to characterize the tumour on a metabolic and molecular basis [14]. [F-18]-fluorodeoxyglucose PET (18F-FDG PET) has been postulated to be more accurate for determining target volumes for various tumors. Interestingly, imaging of brain tumors with 18F-FDG PET was the first oncologic application of PET [15]. Goldman et al. determined that areas of increased 18F-FDG PET activity corresponded well to biopsy-proven anaplastic tumor [16]. Likewise, high PET uptake in a previously known low-grade tumor suggests anaplastic transformation [17]. FDG uptake correlates with tumor grade and aggressiveness, and the level of FDG uptake in primary brain tumors can predict survival as well [16-18]. Alavi et al. found that18F-FDG PET was predictive of survival in high-grade gliomas, with patients having hypermetabolic lesions surviving a mean of 7 months, versus 19 months for patients with hypometabolic lesions [19].

Recently, studies have demonstrated some limitations of 18F-FDG PET while using them for studying brain tumors [20]. Because of the high rate of physiologic glucose metabolism in brain tissue, the delineation of tumors with modest increases in glucose metabolism (low-grade and some recurrent high-grade tumors) is difficult, leading to a decreased specificity [20]. It is not clear which threshold value for the tumor/normal tissue should become the reference value for determining tumors although some reports have suggested a threshold value of 1.3 to 1.7 [21]. Another difficulty with 18F-FDG PET is that postsurgical changes around the resection cavity can exhibit tracer uptake because of inflammation or 18F-FDG leakage caused by disrupted blood brain barrier.

In due course of time, various strategies have been developed to overcome these limitations of 18F- FDG PET. Co-registration of PET images with MR images is one such method which improves the performance of $18 \mathrm{~F}-\mathrm{FDG}$ PET, and it is now a standard practice to have the MR images available while interpreting PET data [22]. Because MRI has high sensitivity but poor specificity, it should be studied first as a screening test [22]. A recent study showed that $18 \mathrm{~F}-$ FDG imaging 3-8 hours after tracer injection can improve the distinction between tumor and normal gray matter [23]. It is based on the hypothesis that glucose excretion from the normal brain cells is increased when the interval between 18F-FDG administration and data acquisition is extended. This excretion is higher in normal brain than in tumor cells, so tumor cells retain 18F-FDG for a longer duration resulting in improved tumor delineation on delayed images [15]. This phenomenon is attributed to increased rate of dephosphorylation of FDG-6-phosphate in tumor cells relative to the normal brain at delayed time. Multiple studies are available to assess the utility of $18 \mathrm{~F}-\mathrm{FDG}$ PET in target volume delineation but there is paucity of data on the use of delayed PET for this purpose, mainly from the eastern part of the world. As 18F-FDG PET is now available in India, there is a need to explore its utility in improving outcome of diseases like glioblastoma.

Recently, it has been suggested that amino acid tracers, like 11Cmethionine (11C-MET), 18F-fluoroethyltyrosine (18F-FET), and 3,4dihydroxy-6-(18)F-fluoro-l-phenylalanine (18F-FDOPA) are more useful for imaging brain tumors than 18F-FDG because of thrie higher uptake in tumor tissues compared to normal brain, giving a better contrast between them [24-28]. Among these tracers, 11C- MET has been most widely studied. However, due to its short half-life, its applicability is limited to facilities with on-site cyclotrons. Thus, it has not achieved a widespread utility in neuro-oncology more-so in developing parts of the world.

The current study is a prospective trial using 18F-FDG PET as a metabolic marker of malignant regions while determining target volumes for RT. A comparison of target volumes between those determined by PET and MRI was done followed by correlation between these volumes and the site of subsequent failure. We hypothesised that PET-based target volumes are different from MRIbased volumes and PET identifies additional foci of active tumor which remains unidentified by MRI alone. These foci remaining obscure on MRI may act as the source for subsequent treatment failure. The idea was to quantitatively assess the advantage of PET in terms of better coverage of high-risk volume and decreased risk of recurrence.

\section{Material and Methods}

This study was conducted at a tertiary care hospital from August 2010 through June 2014. Fifteen patients were enrolled in this study after approval from the Institutional Review Board and independent Ethical Committee. The trial was registered with ClinicalTrials.gov, number NCT01083719. Eligibility criteria included histologically confirmed glioblastoma, age above 18 years, Eastern Cooperative Oncology Group (ECOG) performance status of 0-3. Exclusion criteria included multifocal or recurrent gliomas, and history of diabetes, contrast allergy or prior RT. Informed consent was obtained from each patient before enrolment. Thereafter, each patient underwent clinical work-up and routine blood investigations. Patient underwent thermoplastic cast fabrication for immobilization before simulation scans.

PET-CT was performed on a 64 slice lutetium oxyorthosilicate (LSO) based scanner (Siemens Biograph mCT). The patients fasted for at least 4 hours before the study. After blood glucose measurement (glucose between $60-150 \mathrm{mg} / \mathrm{dL}$ ), $185-190 \mathrm{MBq}$ of $18 \mathrm{~F}-\mathrm{FDG}$ was injected. The dose of F-18 FDG was recorded at the time of injection for estimating standard uptake value (SUV). 18F-FDG was procured from source as unit doses having radiochemical purity in excess of 95\%. After injection, patients were instructed to wait quietly for about 30 minutes in an isolated room. Patients' head was immobilized in cast and scannig was performed in supine position after placing the fiducial markers. The PET images were acquired in a 3-dimensional single bed position for 10 minutes using corrected output image (TrueXHD PET). Emission data was corrected for attenuation, scatter, and random coincidence and reconstructed iteratively using guassian filter with 3 iteration and 24 subsets. Image size was kept at 400 with axial resolution of $2 \mathrm{~mm}$.

Contrast CT was obtained in the same position using field of view (FOV) of $300 \mathrm{~mm}$ using non-iodinated contrast (Omnipaque) 350 
Citation: Takeda K, Dobashi S, Komori S, Chida K, Kadoya N, et al. (2014) A Comparison of Fluro-Deoxy Glucose-Positron Emission Tomography (FDG-PET) Versus Magnetic Resonance (MR) Based Target Volume Delineation in Post-operative Glioblastoma. J Nucl Med Radiat Ther 6: 205. doi:10.4172/2155-9619.1000205

Page 3 of 10

$\mathrm{mg} / 100 \mathrm{ml}$ as per patient's weight. Simultaneous reconstruction was done at $3 \mathrm{~mm}$ using filter H195 low dose for ECT.

Delayed PET scan was acquired at 180-360 minutes [22] after radio tracer injection keeping all the parameters same as in the initial scan. The PET images were fused with attenuation corrected CT.

MRI was performed in supine position using 3-Tesla equipment (Magnetron Verio, Seimens). Images were acquired using standard head coil with rigid immobilization. Axial T1-weighted sequences (matrix size, 256x320; FOV, $250 \mathrm{~mm}$ ) were obtained using gadolinium based contrast medium. The images were acquired from vertex to foramen magnum at $3-\mathrm{mm}$ slice thickness without gap. The T2 weighted images were acquired with a 256x320 matrix and $250 \mathrm{~mm}$ FOV.

The acquired images (CT, MRI and PET) were transferred to FOCAL ${ }^{\star}$ SIM v.4.64 (Elekta, Kungstensgatan, Sweden) contouring platform via DICOM (digital imaging and communications in medicine). Simulation CT scan was fused with T1W MRI and delayed PET using FOCAL SIM. It uses rigid registration using "Auto Fusion" algorithm to optimally register corresponding anatomical details in two and three-dimensional study sets of the same patient's anatomy, using a volume of interest function limited to the region of the tumor. The registration criterion is mutual information, i.e. a measure of the statistical similarity of the overlapping data. The "best registration" is the transformation resulting from an optimization process that gives the maximum value of the mutual information.

Target delineation was performed by the radiation oncologist and verified by another radiation oncologist, a nuclear physician and a radiologist. Three set of volumes were contoured as follows:

MRI-based volumes (for treating patient) by fusing planning CT and T1W MRI 1).

GTV-MR: It included resection cavity with residual tumor (Figure
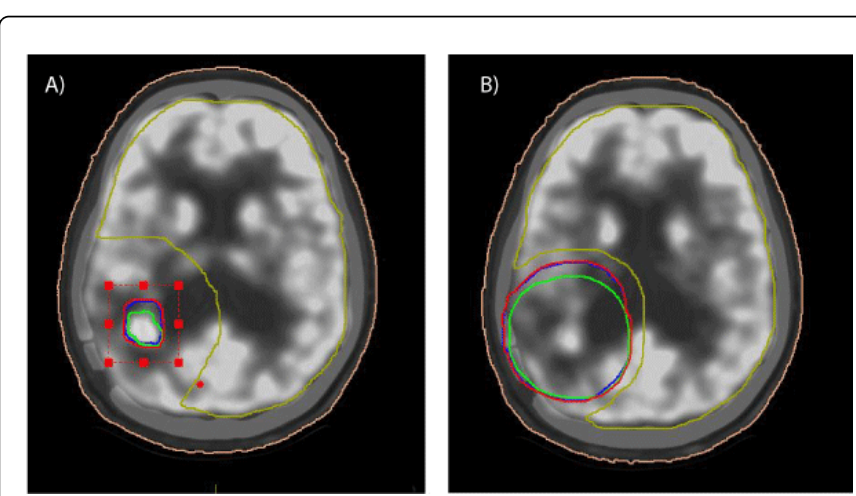

Figure 1: a: Delineation of Gross Tumor Volumes, GTV-MR (Blue), GTV-PET (Green), and GTV-X (Red) on fused image. The GTVPET is inside GTV-MR yielding CI of 0.05 . b: Delineation of respective Clinical Target Volumes, CTV-MR (Blue), CTV-PET (Green), and CTV-X (Red) in the same patient yielding CI of 0.38 .

CTV-MR: GTV-MR plus a $2 \mathrm{~cm}$ margin all around. The margin was reduced to $5 \mathrm{~mm}$ around anatomical barriers such as skull, ventricles, falx etc and to allow for sparing optic nerve/chiasma, if indicated [29] (Figure 2).
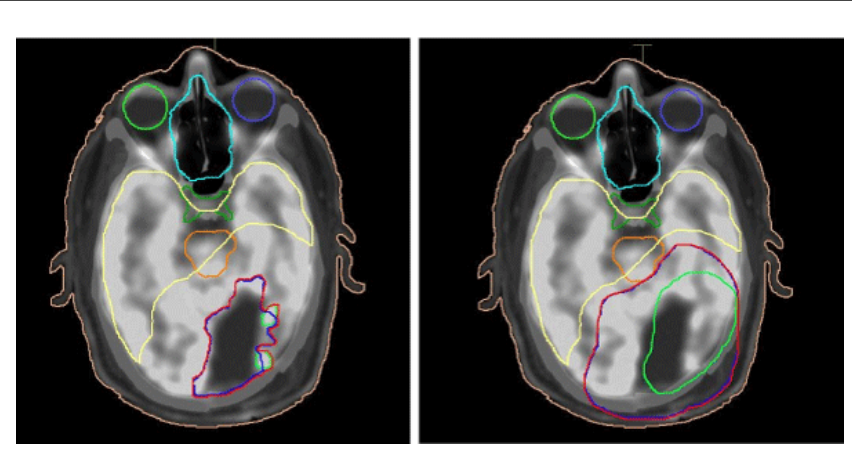

Figure 2: a: Delineation of Gross Tumor Volumes, GTV-MR (Blue), GTV-PET (Green), and GTV-X (Red). The GTV-PET lies partially outside GTV-MR yielding a lower CI of 0.03. b: Delineation of respective Clinical Target Volumes, CTV-MR (Blue), CTV-PET (Green), and CTV-X (Red) in the same patient yielding CI of 0.16.

PTV-MR: CTV-MR plus $5 \mathrm{~mm}$ margin all around [29].

PET-based volumes (for experimental purpose) by fusing planning $\mathrm{CT}$ and delayed PET:

GTV-PET: Marked as metabolically active tumor volume (MATV) which was contoured using two criteria: a) presence of greater uptake of 18F-FDG than normal white matter (Figure 1). To facilitate this, images were displayed with standard gray-scale windowing with a threshold of 1.5 to normal white matter uptake levels [21] and b) presence of any abnormal 18F-FDG uptake within and adjacent to abnormal region on MRI. Elevated FDG uptake in normal appearing area on MRI away from affected region was considered to be functional rather than tumor.

For lesions with a cold center on PET, contours were drawn to include only the outer rim of increased uptake and to exclude areas with normal uptake. For separate lesions with non-contiguous areas of FDG uptake, non-contiguous contours were drawn, and the tumor volume was taken as sum of these non-contiguous contours.

CTV-PET: GTV-PET plus a $2 \mathrm{~cm}$ margin all around respecting anatomical barriers (Figure 2).

PTV-PET: CTV-PET plus $5 \mathrm{~mm}$ margin all around.

Combined volumes (for experimental purpose) by combining PETand MRI-based volumes

GTV-X: It was the volume obtained by combining GTV-MR and GTV-PET and represented the abnormal area using combined modality (Figure 1).

CTV-X: Marked as $2 \mathrm{~cm}$ isotropic expansions of GTV-X, cropped to exclude any portion lying outside the brain [8]. It represented the volume that would have been treated by using combined modality (Figure 2).

PTV-X: CTV-X plus $5 \mathrm{~mm}$ margin.

PTVs were not considered for volumetric analysis as PTV was just a geometric expansion of CTV and reflected CTV itself with no added information. 
Citation: Takeda K, Dobashi S, Komori S, Chida K, Kadoya N, et al. (2014) A Comparison of Fluro-Deoxy Glucose-Positron Emission Tomography (FDG-PET) Versus Magnetic Resonance (MR) Based Target Volume Delineation in Post-operative Glioblastoma. J Nucl Med Radiat Ther 6: 205. doi:10.4172/2155-9619.1000205

Page 4 of 10

\section{Correlation between PET and MRI Volumes}

PET based volumes (GTV-PET and CTV-PET) were compared with MRI-based volumes (GTV-MR and CTV-MR) by using a simple ratio between respective volumes.

Volume locations were assessed and the degree of overlap between volumes was determined by calculating Concordance Index (CI) as $\mathrm{CI}=[(\mathrm{M}+\mathrm{P}) / \mathrm{X}]-1$, where $\mathrm{M}$ is the MR-based volume, $\mathrm{P}$ is PET-based volume, and $\mathrm{X}$ is the combined volume encompassing PET and MRI abnormalities.

Complete concordance between $\mathrm{P}, \mathrm{M}$, and $\mathrm{X}$, would yield a CI of 1 i.e., $[(1+1) / 1]-1=1$. Conversely, complete non-concordance would yield a CI of 0 (i.e., volume $\mathrm{P}$ and $\mathrm{M}$ would be entirely separate, and thus $[(1+1) / 2)]-1=0$. For all other possible scenarios, the CI would lie between 0 and 1 , with higher values representing greater concordance.

\section{Primary Treatment and Follow-up}

Primary treatment included surgery, RT with concurrent and adjuvant TMZ. Patients were treated using IMRT (image guided radiotherapy) to MRI-based target volumes. The dose prescribed to PTV-MR was 5700-6000 cGy in 27-30 fractions with five fractions delivered per week with concurrent TMZ ( $75 \mathrm{mg} / \mathrm{m} 2$ daily) from start till last day of RT. Treatment planning was performed using Monaco ${ }^{\circ}$ v. 3.0 (Elekta, Crawley, UK) planning system.

During RT patients were reviewed weekly with clinical examination and complete heamogram for assessing treatment toxicity as per Radiation Therapy Oncology Group (RTOG) criteria. After RT completion, patients were followed-up after one month with blood investigations and contrast MRI Brain. They were started on adjuvant TMZ (150-200 mg/m2/day) for five days every 28 days for six cycles. An MRI was repeated after third and sixth cycle for response assessment. After six cycles, complete responders were followed up six monthly with contrast MRI brain. In other cases, treatment beyond six cycles was individualized as per the tumor board decision and institutional protocol. In addition, follow-up over phone was continued in case the patient missed his due visits for any reason and this telephonic contact was documented as follow-up. Time to recurrence, last follow-up or overall survival was measured from the date of surgery.

\section{Definition of Response and Analysis of Recurrent/ Progressive Cases}

Treatment response was defined as per Mcdonald criteria [30]. Response to primary treatment was assessed on MRI after one month of completing adjuvant TMZ.

Complete response (CR) was defined as disappearance of all enhancing disease with stable or improving neurologic examination, no new lesion and no increase in steroid dose.

Partial response $(\mathrm{PR})$ required all of following: $>/=50 \%$ reduction in sum of products of perpendicular diameters of enhancing lesions, no new lesion, stable/reduced steroid requirement, stable or improved clinically

Stable disease (SD) included presence of condition not qualifying for $\mathrm{CR}, \mathrm{PR}$ or $\mathrm{PD}$, and stable clinically.
Progressive disease (PD) was defined as greater than $25 \%$ increase in the sum of the product of the perpendicular diameters of enhancing lesions, appearance of any new lesion or clinical worsening.

MRI changes occurring 1-3 months following RT in the absence of clinical symptoms or increased need for steroids were followed closely to rule-out pseudo-progression [31]. If subsequent imaging proved progression, the date of progression was the date of scan which first raised this issue [30].

Recurrence was defined as appearance of new lesion in cases showing CR or a separate lesion in those showing PR or SD. Recurrence free survival (RFS) was calculated from the date of diagnosis to the date of MRI suggesting recurrence. At recurrence, salvage treatment was offered in an individualised manner according to the clinical situation and physician's discretion.

In recurrent cases, the MRI demonstrating recurrence was coregistered with the initial planning $\mathrm{CT}$ containing the previously delineated target volumes. A recurrent volume $(\mathrm{rVol})$ was marked by a neuro-radiologist and a radiation oncologist as the current contrastenhancing region on T1W MRI. The overlapping volume between rVol and CTV-MR, CTV-PET and CTV-X was calculated as a proportion of $\mathrm{rVol}$ lying within the respective CTV. This area of overlap signified the proportion of recurrent volume that would have been covered in the respective CTV.

\section{Statistics}

The primary objective was to assess the volumes delineated using MRI, PET and combined modality and to quantify their mutual agreement with each other. The secondary objectives were to assess overall survival, overall follow-up and recurrence free survival. SPSS v. 12.0 (SPSS Inc., Chicago, IL) software for Windows (Microsoft, Redmond, CA) was used for statistical analysis. Differences in the volume of the consensus MRI, PET, and MRI-PET based contours were quantified using Concordance Index and were statistically tested using 2 -sided paired t-tests (alpha $=0.05$ ). A p-value of 0.05 was considered as significant.

\section{Observation and Results}

\section{Patient characteristics and outcomes}

All 15 enrolled patients completed the study with no refusals or drop outs. The mean age at diagnosis was 52 years with male: female ratio of 2:1 (Table 1).

All patients underwent maximal resection with one-third achieving gross total resection and others achieving near total resection of tumor. $\mathrm{O}(6)$-methylguanine-DNA methyltransferase (MGMT) methylation study was done in only six patients, out of whom half were found to carry methylated promoter region.

These were the patients who were enrolled once MGMT testing became a standard departmental protocol.

Radiotherapy was started after an average interval of 22 days from surgery. Median RT dose was 6000 cGy over six weeks and there were no unplanned gap of more than a week in any patient.

All patients could continue their TMZ during RT without any unplanned gap except one patient whose TMZ was withheld for a week during RT due to skin rash which was later attributed to 
Citation: Takeda K, Dobashi S, Komori S, Chida K, Kadoya N, et al. (2014) A Comparison of Fluro-Deoxy Glucose-Positron Emission Tomography (FDG-PET) Versus Magnetic Resonance (MR) Based Target Volume Delineation in Post-operative Glioblastoma. J Nucl Med Radiat Ther 6: 205. doi:10.4172/2155-9619.1000205

Page 5 of 10

phenytoin and subsided once phenytoin was replaced with levetiracetam.
A median of six cycles of adjuvant TMZ were received by patients with no skin or haemotologic toxicity beyond grade II in any of them.

\begin{tabular}{|c|c|}
\hline Characteristics & \\
\hline \multicolumn{2}{|l|}{ Age in years $(n=15)$} \\
\hline Mean & 52.8 \\
\hline Median & 54 \\
\hline Range & $18-70$ \\
\hline \multicolumn{2}{|l|}{$\operatorname{Sex}(n=15)$} \\
\hline Male & 10 \\
\hline Female & 5 \\
\hline \multicolumn{2}{|l|}{ Dose (in cGy) } \\
\hline Mean & 6000 \\
\hline Median & 6000 \\
\hline Median number of adjuvant TMZ cycles & 6 \\
\hline \multicolumn{2}{|l|}{ MGMT status $(n=6)$} \\
\hline Methylated & 3 \\
\hline Unmethylated & 3 \\
\hline \multicolumn{2}{|l|}{ Type of surgery $(n=15)$} \\
\hline Gross total resection & 05 \\
\hline Near total resection & 10 \\
\hline \multicolumn{2}{|c|}{ Gap between surgery and starting RT (in days) } \\
\hline Mean & 22 \\
\hline Median & 19 \\
\hline Range & $14-32$ \\
\hline \multicolumn{2}{|l|}{ Skin toxicity ( $n=15)$} \\
\hline Grade 0 & 00 \\
\hline Grade 1 & 14 \\
\hline Grade 2 & 01 \\
\hline \multicolumn{2}{|l|}{ Haematologic toxicity $(n=15)$} \\
\hline Grade 0 & 07 \\
\hline Grade 1 & 05 \\
\hline Grade 2 & 03 \\
\hline
\end{tabular}

Table 1: Demographic and treatment characteristics.

\section{Volumetric analysis}

Volumetric analysis was carried out in all 15 patients as per protocol (Table 2). Mean GTV-MR was $84.4 \mathrm{cc}$ while GTV-PET was $11 \mathrm{cc}$, which was only $13 \%$ of GTV- MR and was significantly smaller than GTV-MR ( $\mathrm{p}=0.001)$. At the same time, mean GTV-X was $89.2 \mathrm{cc}$ and was $6 \%$ larger than GTV-MR, the difference between the two was non-significant $(\mathrm{p}=0.86)$.
Thus, PET-based GTV produced a minimal change when combined with MRI volume. In majority of cases, increased PET activity appeared within the region of contrast-enhancement on MRI. However, there were cases where GTV-PET extended beyond GTVMR. In two cases, there was no PET uptake at all and thus GTV-PET was zero. The mean CI for GTVs was 0.10 which signifies that only $10 \%$ volume of GTV-X was overlapping between two modalities. It is expected because of the minimal contribution of GTV-PET to GTV-X. 
Citation: Takeda K, Dobashi S, Komori S, Chida K, Kadoya N, et al. (2014) A Comparison of Fluro-Deoxy Glucose-Positron Emission Tomography (FDG-PET) Versus Magnetic Resonance (MR) Based Target Volume Delineation in Post-operative Glioblastoma. J Nucl Med Radiat Ther 6: 205. doi:10.4172/2155-9619.1000205

Page 6 of 10

Mean CTV-MR was $334.2 \mathrm{cc}$ while CTV-PET was $130.2 \mathrm{cc}$ (39\% of CTV-MR). Again, the PET-based volume was significantly smaller than the MRI-based volume ( $\mathrm{p}=0.00)$. Mean CTV-X was $363 \mathrm{cc}$ and was just $8.6 \%$ larger than CTV-MR and the difference between them was non-significant $(\mathrm{p}=0.60)$. Thus, CTV-PET produced a minimal overall change when combined with CTV-MR to generate CTV-X.

\begin{tabular}{|l|l|l|l|l|l|l|l|l|}
\hline & GTV MR & GTV PET & GTV X & CI (GTV) & CTV MR & CTV PET & CTV X & CI (CTV) \\
\hline Number of patients & 15 & 15 & 15 & 15 & 15 & 15 & 15 & 15 \\
\hline Mean & 84.41 & 11.04 & 89.22 & 0.10 & 334.26 & 130.24 & 363.06 & 0.35 \\
\hline Median & 64.33 & 4.72 & 64.33 & 0.04 & 283.64 & 117.88 & 330.19 & 0.35 \\
\hline Range & $26.55-318.43$ & $0-30.35$ & $31.78-318.43$ & $0-0.24$ & $123.3-714.85$ & $0-295.28$ & $128.1-735.32$ & $0-0.75$ \\
\hline
\end{tabular}

Table 2: Volumetric analysis of different target volumes (in cc).

\section{Treatment response and recurrence pattern}

Disease response could be assessed in 14 patients. In one patient, no follow-up scans were done hence response remained unknown. Overall, one-third patients achieved CR with primary treatment while one-third developed progressive disease (Table 3).

All patients were analyzed for patterns of failure. During follow up, 10 patients developed recurrence by clinical and radiological criteria.

\begin{tabular}{|c|c|}
\hline Characteristic & Numbers (\%) \\
\hline \multicolumn{2}{|l|}{ Response to primary treatment $(n=15)$} \\
\hline Complete response & $05(33)$ \\
\hline Partial response & $04(27)$ \\
\hline Stable disease & $00(00)$ \\
\hline Progressive disease & $05(33)$ \\
\hline Unknown & $01(07)$ \\
\hline Total no. of recurrences & 10 \\
\hline \multicolumn{2}{|l|}{ Recurrent volume in cc $(n=10)$} \\
\hline Mean & 18.57 \\
\hline Median & 13.12 \\
\hline Range & $2.17-68.37$ \\
\hline \multicolumn{2}{|l|}{ Time to recurrence in months $(n=10)$} \\
\hline Mean & 8.6 \\
\hline Median & 7.5 \\
\hline Range & $2.8-15.0$ \\
\hline \multicolumn{2}{|l|}{ Recurrence w.r.t. primary response } \\
\hline Recurrence in complete responders & $60 \%$ \\
\hline Recurrence in partial responders & $75 \%$ \\
\hline Recurrence on progressive cases & $80 \%$ \\
\hline
\end{tabular}

Recurrence was histologically confirmed in only two cases where resurgery was done. All other cases were labelled as 'recurrent' on the basis of clinical and MRI findings. The mean RFS for overall group was 11 months while it was 8.6 months for patients who experienced a recurrence. The recurrence pattern correlated with the initial response to primary treatment as only three out of five complete responders, while four out of five patients with PD developed recurrence.
The mean CI for CTVs was 0.35 which is modest and signifies that $35 \%$ of the combined volume (CTV-X) was overlapping between the two modalities. It is expected because, a) CTV-PET contributed more to CTV-X as compared to respective GTVs and, b) the $2 \mathrm{~cm}$ expansion of the overlapping GTVs resulted in higher overlapping of CTVs. 
Citation: Takeda K, Dobashi S, Komori S, Chida K, Kadoya N, et al. (2014) A Comparison of Fluro-Deoxy Glucose-Positron Emission Tomography (FDG-PET) Versus Magnetic Resonance (MR) Based Target Volume Delineation in Post-operative Glioblastoma. J Nucl Med Radiat Ther 6: 205. doi:10.4172/2155-9619.1000205

Page 7 of 10

\begin{tabular}{|l|l|}
\hline Mean & \multicolumn{2}{l|}{11.1} \\
\hline Median & \multicolumn{2}{l|}{9.5} \\
\hline Patients with recurrence $(\mathbf{n}=\mathbf{1 0})$ & 8.6 \\
\hline Mean & 7.5 \\
\hline Median & \multicolumn{2}{|l|}{} \\
\hline Patients without recurrence $(\mathbf{n}=\mathbf{5})$ & 16 \\
\hline Mean & 13.2 \\
\hline Median & \multicolumn{2}{|l|}{} \\
\hline
\end{tabular}

Table 3: Response to primary treatment and recurrence pattern.

\section{Volume and location of recurrent disease}

The mean recurrent volume ( $\mathrm{rVol})$ was $18.5 \mathrm{cc}$ (Table 4) which was significantly smaller than GTV-MR. In one patient, two recurrent lesions were seen, which were counted as two separate lesions. One of these lesions was completely away from all three initial CTVs.

\begin{tabular}{|l|l|l|l|}
\hline & CTV MR (\%)[n=11] & CTV PET (\%)[n=10] & CTV X (\%)[n=11] \\
\hline Mean & 73.7 & 57.7 & 81.7 \\
\hline Median & 100 & 69 & 100 \\
\hline Range & $0-100$ & $0-100$ & $0-100$ \\
\hline
\end{tabular}

Table 4: Overlap of recurrent volume with different CTVs.

On an average, $73.7 \%$ of $\mathrm{rVol}$ was overlapping with CTV-MR $(\mathrm{n}=11$, as two lesions in a patient) while $81.7 \%$ of $\mathrm{rVol}$ was overlapping with CTV-X. Thus, coverage of recurrent volume was higher with CTV-X as compared to CTV-MR, although the difference was statistically non-significant $(\mathrm{p}=0.49)$. It indicates that if CTV-X would have been used for initial treatment, higher proportion of 'at risk' volume would have been treated.

\begin{tabular}{|c|c|}
\hline Characteristic & Numbers \\
\hline \multicolumn{2}{|l|}{ Salvage treatment $(n=10)$} \\
\hline Chemotherapy only & 03 \\
\hline RT only & 01 \\
\hline Surgery + Chemotherapy & 01 \\
\hline RT + Chemotherapy & 03 \\
\hline Surgery+RT+Chemotherapy & 01 \\
\hline None & 01 \\
\hline \multicolumn{2}{|c|}{ Follow-up after salvage treatment $(n=9)$ in months } \\
\hline Mean & 11.9 \\
\hline Median & 9.7 \\
\hline Range & $1-28$ \\
\hline
\end{tabular}

\section{Salvage Treatment}

Out of the 10 recurrent cases, nine underwent salvage treatment (Table 5). Four patients received re-irradiation as a part of salvage treatment where treatment was given to the recurrent lesion with strict margins using stereotactic techniques. Stereotactic RT was delivered using linear accelerator as well as Cyberknife.

In seven cases, chemotherapy was given as a part of salvage treatment, where five patients received metronomic schedule of TMZ and other two received bevacizumab based chemotherapy. One young patient underwent multiple salvage treatments during his follow-up of 23.4 month including re-irradiation twice (one SFRT and one SRS), two salvage surgeries and bevacizumab based chemotherapy. He survived 17 months following recurrence. Out of two patients who underwent re-surgery, one patient also underwent intra-operative Carmustine wafer placement. Response to salvage treatment was not recorded. Average follow-up after starting salvage treatment was 11.9 months.

Table 5: Salvage treatment and follow-up. 
Citation: Takeda K, Dobashi S, Komori S, Chida K, Kadoya N, et al. (2014) A Comparison of Fluro-Deoxy Glucose-Positron Emission Tomography (FDG-PET) Versus Magnetic Resonance (MR) Based Target Volume Delineation in Post-operative Glioblastoma. J Nucl Med Radiat Ther 6: 205. doi:10.4172/2155-9619.1000205

Page 8 of 10

\section{Survival and follow up}

Last data analysis was done in June 2014, with a follow-up of 22 months for the last recruited patient (Table 6). Median follow-up was 16.6 months for the overall group and 26.1 months for the patients surviving at last follow-up.
Overall, four patients were alive at more than two years of followup, with two of them having disease-free status. These two patients had RFS of 29.6 and 22.7 months, both them had CR to primary treatment. Overall 1- and 2-year survival was $80 \%$ and $20 \%$ respectively. If last follow-up is taken as an event, the median survival of the whole group was 16.6 months.

\begin{tabular}{|c|c|}
\hline \multicolumn{2}{|l|}{ Characteristic } \\
\hline Median survival $(n=15)$ & 16.6 months \\
\hline \multicolumn{2}{|l|}{ Overall survival $(n=15)$} \\
\hline 12-month & $80 \%$ \\
\hline 18-month & $46.6 \%$ \\
\hline 24-month & $20 \%$ \\
\hline \multicolumn{2}{|c|}{ Follow up of overall group $(n=15)$ in months } \\
\hline Mean & 19.0 \\
\hline Median & 16.6 \\
\hline Range & $4.8-42.8$ \\
\hline \multicolumn{2}{|c|}{ Follow-up of surviving patients $(n=4)$ in months } \\
\hline Mean & 29.3 \\
\hline Median & 26.1 \\
\hline Range & $22-42.8$ \\
\hline \multicolumn{2}{|c|}{ Status at last follow-up $(n=15)$} \\
\hline Alive with disease & $02(13.3 \%)$ \\
\hline Alive without disease & $02(13.3 \%)$ \\
\hline Died & $11(73.3 \%)$ \\
\hline
\end{tabular}

Table 6: Overall survival and follow-up.

\section{Discussion}

This study is first of its kind to be conducted in India involving patients of GBM. The overall idea of this study was to assess the potential utility of $18 \mathrm{~F}$-FDG PET in identifying regions which are at high risk for recurrence while delineating target volumes for RT. We hypothesized that if PET is used in addition to MRI for delineating such regions, there would be lesser marginal failures.

Although, statistically non-significant, we found a better coverage of recurrent lesions when PET and MRI were used together to delineate CTV-X. Moreover, this better coverage of 'at risk' region was achieved by adding a non-significant volume of normal brain to CTV$\mathrm{MR}$, as CTV-X was just $8.6 \%$ larger than CTV-MR.

The mean PET-based volumes were consistently smaller than MRbased volumes with low CI. The low CI verified the qualitative observation that regions of FDG uptake overlapped some but not all regions of enhancement on MRI and extended outside GTV-MR in some cases. Such discordance has also been described by other investigators and may be of prognostic significance [19]. In one such study, $87 \%$ of patients were found to have discrepancies between the gadolinium-enhancing volume and areas of increased PET activity [32]. However, that study did not include data on subsequent failures, so it remained unclear whether PET activity would be important in identifying regions at high risk for progression [32]. Our study has tried to answer these queries. While studies document the level of $18 \mathrm{~F}$ FDG uptake in tumor to be predictive of survival, there is limited literature to exactly show that the $18 \mathrm{~F}-\mathrm{FDG}$-avid volume and its spatial distribution may also predict outcome.

Although, 18F-FDG PET volumes were an indicator of area at risk for geographic miss, the rate of false-negative PET studies is worrisome as PET is not an absolute indicator of absent disease. Moreover, it is unclear whether the additional volume delineated with PET should be used for overall treatment or as a boost. Gross et al. incorporated FDG-PET results with MRI for delineation of the 3dimensional 60 Gy treatment volumes for 18 cases of high-grade glioma. Their reported median survival of 44 weeks showed no improvement compared with other studies. They concluded that FDGPET did not add useful information for conventional treatment planning but suggested that it might prove useful to define a boost volume [10]. Although, few centers are exploring the incorporation of FDG-PET volumes for a simultaneous integrated boost in IMRT and for planning SRS target volumes [33], these approaches need to demonstrate efficacy before they are applied in clinical practice. 
Citation: Takeda K, Dobashi S, Komori S, Chida K, Kadoya N, et al. (2014) A Comparison of Fluro-Deoxy Glucose-Positron Emission Tomography (FDG-PET) Versus Magnetic Resonance (MR) Based Target Volume Delineation in Post-operative Glioblastoma. J Nucl Med Radiat Ther 6: 205. doi:10.4172/2155-9619.1000205

Page 9 of 10

Our study shows that RT protocols based on 18F-FDGPET imaging are feasible and suitable for patients in developing countries like India where other amino acid based PET are currently unavailable for routine clinical use. Although, 18FDG-PET has its limitations in delineating brain lesions, it can be overcome upto an extent by using delayed scans as delineation of gliomas from both gray matter and white matter improves both visually and by SUV analysis on delayed PET [23] at the cost of extra waiting time for the delayed scan. In future, similar strategies could be applied to identify regions of hypoxia, proliferation, protein synthesis, or membrane biosynthesis.

Although combined volume (CTV-X) showed potential for covering increased proportion of 'at risk' volume, treatment using PET-based volumes may not be fool-proof method as one patient in this study developed a recurrent lesion even outside CTV-X. Thus, there is a need to balance the margins used for delineating CTVs and the acceptable toxicity to normal brain.

The age and sex distribution is comparable to literature, although the median age at diagnosis seems lesser ( 52 years) than reported in literature [2]. One specific feature of our study was no denial, drop outs or loss of follow-up which may be contributed to the stringent and streamlined enrolment, treatment and follow-up process where mutual communication with patients using telephone and e-mails was encouraged to keep them informed and updated regarding their status. Our median OS (16.6 months) is a shade superior compared to 14.6 months reported by Stupp et al. [8]. However, the 2 -year OS of $20 \%$ in our study is mildly inferior to other similar study [34] but it may improve in future as last two recruited patients complete their 2-year follow- up.

This study shows that Indian patients tolerates concurrent RT with TMZ well, as there were no severe skin or haematological toxicity reported in any of our patients. Moreover, all patients completed their primary treatment without any unplanned gap in treatment.

Metronomic dose of TMZ requires special mention as it was the commonest salvage treatment in inoperable patients at recurrence. It seems to be a feasible, acceptable and well tolerated salvage treatment showing satisfactory results. The patient having longest follow-up (42.8 months) has been continuing his metronomic TMZ for 28 months following recurrence. Similarly, re-irradiation using stereotactic radiotherapy was also well tolerated without any untoward toxicity. Thus, in properly selected patients, re-irradiation followed by metronomic TMZ may be an ideal approach as patient can continue this treatment while at home and without compromising their quality of life. This strategy needs further exploration in future studies.

Although, MGMT methylation status was analyzed in six patients, no formal analysis could be done due to limited numbers for deriving any meaningful conclusion. This widely studied prognostic and predictive aspect in Western literature needs to be studied in Indian patients in future studies.

One limitation of this study is the small number of participants. It was a pilot study where the purpose was to evaluate whether delayed PET might be feasible and useful for treatment volume delineation in glioblastoma patients and we could get an appropriate answer. The correlation we found between the location of increased PET activity and the subsequent failure provides justification for same and warrants additional work in this direction. Another potential limitation is limited follow-up for the patients enrolled at the end, although that factor may be addressed by continuing the data collection and reporting in future.
Further research is warranted to determine whether treatment planning using novel biological imaging can improve outcome for high-grade glioma. In future, it seems likely that the complementary information obtained with multiple imaging modalities will lead to more efficient delineation of target volumes but till then F18FDG-PET may serve this purpose.

\section{Conclusion}

- Delayed 18-FDG PET is a feasible method to provide additional information for delineating target volumes for RT planning in glioblastoma patients.

- Inclusion of delayed FDG-PET based abnormality while delineating the target volumes for GBM leads to a marginal and insignificant increase in target volume.

- There is better coverage of 'high risk' volume using combined volume for radiation.

- Treating this common volume (CTV X) instead of CTV MR may be beneficial in avoiding marginal recurrences in future.

- Future trials with more number of patients and a longer follow-up may further augment our findings

\section{References}

1. http://www.cbtrus.org.

2. Ohgaki H, Kleihues P (2005) Population-based studies on incidence, survival rates, and genetic alterations in astrocytic and oligodendroglial gliomas. J Neuropathol Exp Neurol 64: 479-489.

3. Aydin H, Sillenberg I, von Lieven H (2001) Patterns of failure following CT-based 3-D irradiation for malignant glioma. Strahlenther Onkol 177: 424-431.

4. Garden AS, Maor MH, Yung WK, Bruner JM, Woo SY, et al. (1991) Outcome and patterns of failure following limited-volume irradiation for malignant astrocytomas. Radiother Oncol 20: 99-110.

5. Wallner KE, Galicich JH, Krol G, Arbit E, Malkin MG (1989) Patterns of failure following treatment for glioblastoma multiforme and anaplastic astrocytoma. Int J Radiat Oncol Biol Phys 16: 1405-1409.

6. Chang EL, Akyurek S, Avalos T, Rebueno N, Spicer C, et al. (2007) Evaluation of peritumoral edema in the delineation of radiotherapy clinical target volumes for glioblastoma. Int J Radiat Oncol Biol Phys 68: 144-150.

7. Nelson DF, Diener-West M, Horton J, Chang CH, Schoenfeld D, et al. (1988) Combined modality approach to treatment of malignant gliomas: Re-evaluation of RTOG7401/ECOG 1374 with long-term follow-up. A joint study of the Radiation Therapy Oncology Group and the Eastern Cooperative Oncology Group. Natl Cancer Inst Monogr 6: 279-284.

8. Stupp R, Mason WP, van den Bent MJ, Weller M, Fisher B, et al. (2005) Radiotherapy plus concomitant and adjuvant temozolomide for glioblastoma. N Engl J Med 352: 987-996.

9. Kelly PJ, Daumas-Duport C, Kispert DB, Kall BA, Scheithauer BW, et al. (1987) Imaging-based stereotaxic serial biopsies in untreated intracranial glial neoplasms. J Neurosurg 66: 865-874.

10. Gross MW, Weber WA, Feldmann HJ, Bartenstein P, Schwaiger M, et al. (1998) The value of F-18-fluorodeoxyglucose PET for the 3-D radiation treatment planning of malignant gliomas. Int J Radiat Oncol Biol Phys 41: 989-995.

11. Watling CJ, Lee DH, Macdonald DR, Cairncross JG (1994) Corticosteroid-induced magnetic resonance imaging changes in patients with recurrent malignant glioma. J Clin Oncol 12: 1886-1889.

12. Wen PY, Macdonald DR, Reardon DA, Cloughesy TF, Sorensen AG, et al. (2010) Updated response assessment criteria for high-grade gliomas: response assessment in neuro-oncology working group. J Clin Oncol 28: 1963-1972. 
Citation: Takeda K, Dobashi S, Komori S, Chida K, Kadoya N, et al. (2014) A Comparison of Fluro-Deoxy Glucose-Positron Emission Tomography (FDG-PET) Versus Magnetic Resonance (MR) Based Target Volume Delineation in Post-operative Glioblastoma. J Nucl Med Radiat Ther 6: 205. doi:10.4172/2155-9619.1000205

Page 10 of 10

13. Alexander M. Spence, David A. Mankoff, Mark Muzi (2007) The role of PET in the management of brain tumors. Applied radiology: 8-21.

14. Gambhir SS (2002) Molecular imaging of cancer with positron emission tomography. Nat Rev Cancer 2: 683-693.

15. Chen W (2007) Clinical applications of PET in brain tumors. J Nucl Med 48: $1468-1481$.

16. Goldman S, Levivier M, Pirotte B, Brucher JM, Wikler D, et al. (1996) Regional glucose metabolism and histopathology of gliomas. A study based on positron emission tomography-guided stereotactic biopsy. Cancer 78: 1098-1106.

17. Padma MV, Said S, Jacobs M, Hwang DR, Dunigan K, et al. (2003) Prediction of pathology and survival by FDG PET in gliomas. J Neurooncol 64: 227-237.

18. Barker FG 2nd, Chang SM, Valk PE, Pounds TR, Prados MD (1997) 18 Fluorodeoxyglucose uptake and survival of patients with suspected recurrent malignant glioma. Cancer 79: 115-126.

19. Vlasenko A, Beattie B, Krol G, Blasberg R (2004) Spatial and volumetric relationship between 18F-FDG hypermetabolism and Gd-DTPA enhancement in patients with recurrent glioma [abstract]. J Nucl Med. 41:217P.

20. Ricci PE, Karis JP, Heiserman JE, Fram EK, Bice AN, et al. (1998) Differentiating recurrent tumor from radiation necrosis: time for reevaluation of positron emission tomography? AJNR Am J Neuroradiol 19: 407-413.

21. Hatazawa J, Ishiwata K, Itoh M, Kameyama M, Kubota K, et al. (1989) Quantitative evaluation of L-[methyl-C-11] methionine uptake in tumor using positron emission tomography. J Nucl Med 30: 1809-1813.

22. Wong TZ, Turkington TG, Hawk TC, Coleman RE (2004) PET and brain tumor image fusion. Cancer J 10: 234-242.

23. Spence AM, Muzi M, Mankoff DA, O'Sullivan SF, Link JM, et al. (2004) 18F-FDG PET of gliomas at delayed intervals: improved distinction between tumor and normal gray matter. J Nucl Med 45: 1653-1659.

24. Pirotte B, Goldman S, Massager N, David P, Wikler D, et al. (2004) Comparison of 18F-FDG and 11C-methionine for PET-guided stereotactic brain biopsy of gliomas. J Nucl Med 45: 1293-1298

25. Kracht LW, Miletic H, Busch S, Jacobs AH, Voges J, et al. (2004) Delineation of brain tumor extent with [11C]L-methionine positron emission tomography: local comparison with stereotactic histopathology. Clin Cancer Res 10: 7163-7170.

26. Stadlbauer A, Prante O, Nimsky C, Salomonowitz E, Buchfelder M, et al. (2008) Metabolic imaging of cerebral gliomas: spatial correlation of changes in O-(2-18F-fluoroethyl)-L-tyrosine PET and proton magnetic resonance spectroscopic imaging. J Nucl Med 49: 721-729.

27. Weber DC, Zilli T, Buchegger F, Casanova N, Haller G, et al. [(18)F] fluoroethyltyrosinepositron emission tomography-guided radiotherapy for high-grade glioma. Radiat Oncol 3: 44.

28. Chen W, Silverman DH, Delaloye S, Czernin J, Kamdar N, et al. (2006) 18F-FDOPA PET imaging of brain tumors: comparison study with 18FFDG PET and evaluation of diagnostic accuracy. J Nucl Med 47: 904-911.

29. Chang EL, Akyurek S, Avalos T, Rebueno N, Spicer C, et al. (2007) Evaluation of peritumoral edema in the delineation of radiotherapy clinical target volumes for glioblastoma. Int J Radiat Oncol Biol Phys 68: 144-150.

30. Macdonald DR, Cascino TL, Schold SC Jr, Cairncross JG (1990) Response criteria for phase II studies of supratentorial malignant glioma. J Clin Oncol 8: 1277-1280.

31. Taal W, Brandsma D, de Bruin HG, Bromberg JE, Swaak-Kragten AT, et al. (2008) The incidence of pseudo-progression in a cohort of malignant glioma patients treated with chemo-radiation with temozolomide. J Clin Oncol 113: 405-410.

32. Grosu AL, Weber WA, Riedel E, Jeremic B, Nieder C, et al. (2005) L(methyl-11C) methionine positron emission tomography for target delineation in resected high-grade gliomas before radiotherapy. Int J Radiat Oncol Biol Phys 63: 64-74.

33. Levivier M, Massager N, Wikler D, Lorenzoni J, Ruiz S, et al. (2004) Use of stereotactic PET images in dosimetry planning of radiosurgery for brain tumors: clinical experience and proposed classification. J Nucl Med 45: 1146-1154.

34. Stupp R, Hegi ME, Mason WP, van den Bent MJ, Taphoorn MJ, et al. (2009) Effects of radiotherapy with concomitant and adjuvant temozolomide versus radiotherapy alone on survival in glioblastoma in a randomised phase III study: 5-year analysis of the EORTC-NCIC trial. Lancet Oncol 10: 459-466.
This article was originally published in a special issue, entitled: "Cancer Radiation Therapy", Edited by University of Arkansas for Medical Sciences, USA 